\title{
Efficacy of interspinous device versus surgical decompression in the treatment of lumbar spinal stenosis: a modified network analysis
}

Authors Dean Chou ${ }^{1}$, Darryl Lau ${ }^{2}$, Jeffrey Hermsmeyer ${ }^{3}$, Daniel Norvell ${ }^{3}$

Institutions ${ }^{1}$ University of California, San Francisco, CA, USA

2 University of Michigan Medical School, Ann Arbor, MI, USA

${ }^{3}$ Spectrum Research Inc, Tacoma, WA, USA

\section{ABSTRACT}

Study design: Systematic review using a modified network analysis.

Objectives: To compare the effectiveness and morbidity of interspinous-device placement versus surgical decompression for the treatment of lumbar spinal stenosis.

Summary: Traditionally, the most effective treatment for degenerative lumbar spinal stenosis is through surgical decompression. Recently, interspinous devices have been used in lieu of standard laminectomy.

Methods: A review of the English-language literature was undertaken for articles published between 1970 and March 2010. Electronic databases and reference lists of key articles were searched to identify studies comparing surgical decompression with interspinous-device placement for the treatment of lumbar spinal stenosis. First, studies making the direct comparison (cohort or randomized trials) were searched. Second, randomized controlled trials (RCTs) comparing each treatment to conservative management were searched to allow for an indirect comparison through a modified network analysis approach. Comparison studies involving simultaneous decompression with placement of an interspinous device were not included. Studies that did not have a comparison group were not included since a treatment effect could not be calculated. Two independent reviewers assessed the strength of evidence using the GRADE criteria assessing quality, quantity, and consistency of results. The strengths of evidence for indirect comparisons were downgraded. Disagreements were resolved by consensus.

Results: We identified five studies meeting our inclusion criteria. No RCTs or cohort studies were identified that made the direct comparison of interspinous-device placement with surgical decompression. For the indirect comparison, three RCTs compared surgical decompression to conservative management and two RCTs compared interspinous-device placement to conservative management. There was low evidence supporting greater treatment effects for interspinous-device placement compared to decompression for disability and pain outcomes at 12 months. There was low evidence demonstrating little to no difference in treatment effects between the groups for walking distance and complication rates.

Conclusion: The indirect treatment effect for disability and pain favors the interspinous device compared to decompression. The low evidence suggests that any further research is very likely to have an important impact on the confidence in the estimate of effect and is likely to change the estimate. No significant treatment effect differences were observed for postoperative walking distance improvement or complication rates; however, findings should be considered with caution because of indirect comparisons and short follow-up periods.

This systematic review was funded by AOSpine 


\section{STUDY RATIONALE AND CONTEXT}

Traditionally, the most effective treatment for degenerative lumbar spinal stenosis is through surgical decompression [1]. Recently, interspinous devices have been used in lieu of standard laminectomy. The idea of using a device to distract the spinous processes for symptomatic relief is not new. It was first used in the 1950s but was abandoned secondary to device dislodgement, poor clinical indications, and implant malfunction. It is only recently that interspinous devices are being more popularized with various designs (from static spacers to dynamic devices) composed of an array of materials including allograft, titanium, polyetheretherketone, and elastomeric compounds. The mechanical mechanism by which interspinous devices are purported to treat spinal stenosis is to focally create slight spinal flexion by distraction of the spinous processes. This mimics the position of sitting, which increases the room for the nerves, potentially alleviating pain. Implantation of interspinous devices is a relatively straightforward surgical procedure and can be performed under local anesthesia. However, it is important to clearly identify evidence of whether interspinous devices are able to provide effective treatment and achieve similar goals to surgical decompression for degenerative lumbar spinal stenosis. In addition, the placement of interspinous devices is touted to be less morbid than a standard surgical decompression.

\section{OBJECTIVES}

- To compare the effectiveness of interspinous-device placement versus surgical decompression for the treatment of lumbar spinal stenosis.

- To compare the morbidity of interspinous-device placement versus surgical decompression for the treatment of lumbar spinal stenosis.

\section{MATERIALS AND METHODS}

Study design: Systematic review using a modified network analysis for indirect comparisons.

\section{Sampling:}

- Search: PubMed, Cochrane collaboration database, and National Guideline Clearinghouse databases; bibliographies of key articles.

- Dates searched: 1970 through March 2010.

Inclusion criteria: Patients with lumbar spinal stenosis. Randomized controlled trials (RCTs) and nonrandomized comparison studies. RCTs only for indirect comparisons.

Exclusion criteria: Trauma, significant lumbar instability, previous lumbar spine surgery, spondylolisthesis $>$ grade I and patient younger than 40 years. Comparison studies involving decompression surgery with simultaneous interspinous-device placement. Case series of interspinous-device placement or decompression surgery were not included because of the inability to calculate treatment effects without a comparison group.

Outcomes: Disability, pain, function, and morbidity measures.

Analysis: Changes in outcome scores from baseline to follow-up and complication rates were extracted for all studies. Treatment effects were calculated by comparing change scores between treatment groups. For the studies without a direct comparison between surgical decompression and interspinousdevice placement, a modified network analysis for indirect treatment comparisons was made. The treatment effect was calculated by comparing the treatment effects of decompression versus conservative management to the treatment effects of interspinous-device placement to conservative management. Mean treatment effects between studies were compared. Complications were pooled; however, scores from functional measures were not pooled. Two independent reviewers assessed the strength of evidence using the GRADE criteria assessing quality, quantity, and consistency of results. Quality of evidence was downgraded for indirect comparisons and for small number of studies.

Details about methods can be found in the electronic supplemental material at www.aospine.org/ebsj 


\section{RESULTS}

We identified 97 citations and reviewed 85 abstracts. After abstract review, we excluded 72 studies that did not meet inclusion/exclusion criteria. We reviewed 13 fulltext articles. Eight of these were excluded, leaving five studies meeting our inclusion criteria (Fig 1). No RCTs or cohort studies making the direct comparison were identified; however, one study compared decompression with interspinous-device placement to decompression only [2] and one compared interspinous-device placement to decompression with instrumented fusion [3]; therefore, they were excluded. Three RCTs compared surgical decompression to conservative management and two RCTs compared interspinous-device placement to conservative management. These five studies were selected and used to perform indirect comparisons through a modified network analysis. All studies included subjects who had failed conservative management. Details of each study can be found in Tables 1 and 2.

\section{Description of included studies}

- Treatment effects comparing decompression to conservative management were compared to the treatment effects comparing interspinous-device placement only to conservative management in different RCTs (modified network analysis).

- RCT 1 compared surgical decompression only $(n=50)$ with nonoperative treatment consisting of nonsteroidal antiinflammatory drugs and physical therapy $(n=44)$ [4].

- RCTs 2 and 3 (two studies) were combined as we used data from both studies which included 2- and 4-year follow-ups, respectively. Patients were either assigned to a surgical decompression-only group $(n=138)$ or to a nonoperative group consisting of physical therapy, nonsteroidal antiinflammatory drugs and epidural injections $(n=151)[1,5]$. These studies analyzed data with an intent-to-treat analysis and with an as-treated analysis separately. We summarized the intent-to-treat analysis because the RCT that it is compared to also analyzed data by intent to treat.

- RCTs 4 and 5 (two studies) were combined as we used data from both studies that included 1- and 2-year follow-ups, respectively. Patients were either assigned to an interspinous-device placement group $(n=100)$ or to a nonoperative group consisting of physical therapy, nonsteroidal antiinflammatory drugs, analgesics, and at least one epidural steroid injection $(n=91)[6,7]$.

\section{Disability outcomes comparing interspinous devices to decompression}

The two treatments can be compared indirectly in the RCTs by Zucherman et al $[6,7]$ and Weinstein et al $[1,5]$ since they used the same outcomes measures (SF-36 Physical Function scores, respectively) (Table 3). The 12-month indirect treatment effect was 18.4 (20 versus 1.6) points using the SF-36 Physical Function score [1, 5-7] (Fig 2).

\section{Pain outcomes comparing interspinous-device placement to surgical decompression}

- Differences in pain were observed through indirect comparisons. The RCTs by Weinstein et al $[1,5]$ and Zucherman et al $[6,7]$ measured change in SF-36 bodily pain scores at 12 months. The improvement in pain score was 23 and 31 points for the surgical decompression and interspinous groups, respectively (Table 3). When compared to the conservative groups, the treatment effects were 5.5 and 22 points, respectively. Therefore, the greater pain treatment effect (16.5 points) was observed in the interspinous group (Fig 2).

- Weinstein et al $[1,5]$ administered several other pain measures including a Low Back Pain Bothersome score, Leg Pain Bothersome score, and a Stenosis Bothersome score. When comparing the decompression to conservative management groups, the treatment effects were not statistically significant (Table 2).

Functional outcomes comparing interspinous-device placement to surgical decompression

- The indirect treatment effects for improvement in walking distances comparing decompression (Malmivaara et al [4]) and interspinous (Zucherman et al [6, 7]) groups to conservative groups were $23 \%$ and $25 \%$, respectively (Table 3 and Fig 2).

Safety outcomes comparing interspinous-device placement to surgical decompression

- The two RCTs by Malmivaara et al [4] and Weinstein et al $[1,5]$ reported $27 \%$ and $11.6 \%$ complication rates (perioperative and postoperative complications) in the surgical decompression groups compared to $0 \%$ in the conservative groups at 12 months, respectively. The RCT by Zucherman et al $[6,7]$ reported an $11 \%$ complication rate in the interspinous group compared to $0 \%$ in the conservative group.

- The pooled complication rate for decompression was $15.2 \%$ and the complication rate for the interspinous device was $11 \%\left(\chi^{2} P=.33\right)$ (Fig 2). 
Table 1 Demographics of studies for lumbar spinal stenosis (LSS) treated by decompression or interspinous device.

\begin{tabular}{|c|c|c|c|c|c|}
\hline Reference & Study design & $\begin{array}{l}\text { Study } \\
\text { population }\end{array}$ & Treatment description & Inclusion and exclusion criteria & $\begin{array}{l}\text { Follow-up time, } \\
\% \text { F/U }\end{array}$ \\
\hline 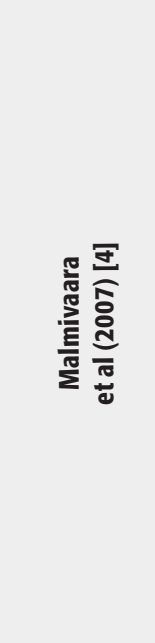 & $\begin{array}{l}\text { Randomized } \\
\text { controlled trial } \\
\text { December } \\
\text { 1997-March } 2001\end{array}$ & $\begin{array}{l}N=94 \\
\text { Decompression } \\
\text { - } n=50 \\
\text { - Age: } 63 \pm 9 y \\
\text { - Male: } 22 \% \\
\text { Nonoperative } \\
\text { treatment } \\
\text { - } n=44 \\
\text { - Age: } 62 \pm 9 y \\
\text { - Male: } 45 \%\end{array}$ & $\begin{array}{l}\text { Decompression: } \\
\text { - Segmental decompression } \\
\text { - Undercutting facetectomy } \\
\text { - Fusion if instability present or } \\
\text { if a risk of instability } \\
\text { Nonoperative: } \\
\text { - Nonsteroidal } \\
\text { antiinflammatory drugs } \\
\text { - Physical therapy consisting of } \\
\text { body posture, trunk } \\
\text { strengthening, stretching } \\
\text { exercise, modalities }\end{array}$ & $\begin{array}{l}\text { Inclusion: } \\
\text { - Clinical and x-ray symptomatic LSS } \\
\text { - Duration of symptoms }>6 \text { mo } \\
\text { - Failed conservative treatment } \\
\text { - Persistent pain without progressive } \\
\text { neurological dysfunction } \\
\text { Exclusion: } \\
\text { - Severe LSS with intractable pain and } \\
\text { progressive neurological dysfunction } \\
\text { - Mild LSS feeble enough to exclude } \\
\text { surgery } \\
\text { - Stenosis not caused by degeneration } \\
\text { - Spondylolytic spondylolisthesis } \\
\text { - Previous lumbar spine surgery } \\
\text { - Diagnosed lumbar herniated disc } \\
\text { - Other spinal disorders } \\
\text { - Severe osteoarthritis causing lower limb } \\
\text { dysfunction }\end{array}$ & $\begin{array}{l}24 \text { mo: } \\
\text { - Decompression: 86\% } \\
\text { - Nonoperative: 91\% } \\
12 \text { mo: } \\
\text { - Decompression: 96\% } \\
\text { - Nonoperative: 93\% } \\
6 \text { mo: } \\
\text { - Decompression: 88\% } \\
\text { - Nonoperative: 93\% } \\
\text { Available for all } \\
\text { follow-ups: } \\
\text { - Decompression: 86\% } \\
\text { - Nonoperative: 84\% }\end{array}$ \\
\hline 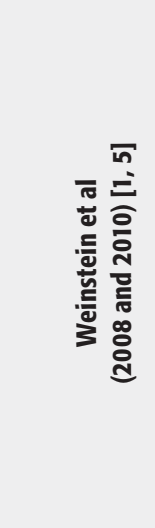 & $\begin{array}{l}\text { Randomized } \\
\text { controlled trial } \\
\text { intent-to-treat } \\
\text { analysis } \\
\text { March } \\
2000 \text { - March } \\
2005\end{array}$ & $\begin{array}{l}\text { N=289 } \\
\text { - Age: } 65.5 \pm 10.5 y \\
\text { - Male: } 62 \% \\
\text { Assigned to } \\
\text { decompression group } \\
\text { - } n=138 \\
\text { - Age: not reported (NR) } \\
\text { - Male: NR } \\
\text { Assigned to } \\
\text { nonoperative treatment } \\
\text { group } \\
\text { - } n=151 \\
\text { - Age: NR } \\
\text { - Male: NR }\end{array}$ & $\begin{array}{l}\text { Decompression: } \\
\text { - Standard posterior } \\
\text { decompression laminectomy } \\
\text { Nonoperative: } \\
\text { - Physical therapy } \\
\text { - Home exercise instruction } \\
\text { - Epidural injections } \\
\text { - Chiropractic care } \\
\text { - Nonsteroidal } \\
\text { antiinflammatory drugs } \\
\text { - Opioid analgesics }\end{array}$ & $\begin{array}{l}\text { Inclusion: } \\
\text { - X-ray confirmation of LSS } \\
\text { - History of neurogenic claudication or } \\
\text { radicular leg symptoms }>12 \text { weeks } \\
\text { - One or more levels } \\
\text { - All patients judged to be surgical } \\
\text { candidates } \\
\text { Exclusion: } \\
\text { - Lumbar instability } \\
\text { - Patients with degenerative } \\
\text { spondylolisthesis studied separately }\end{array}$ & $\begin{array}{l}48 \text { mo: } \\
\text { - Decompression: 67\% } \\
\text { - Nonoperative: 64\% } \\
24 \text { mo: } \\
\text { - Decompression: } 79 \% \\
\text { - Nonoperative: } 75 \% \\
12 \text { mo: } \\
\text { - Decompression: 87\% } \\
\text { - Nonoperative: 83\% } \\
6 \text { mo: } \\
\text { - Decompression: } 87 \% \\
\text { - Nonoperative: } 89 \%\end{array}$ \\
\hline 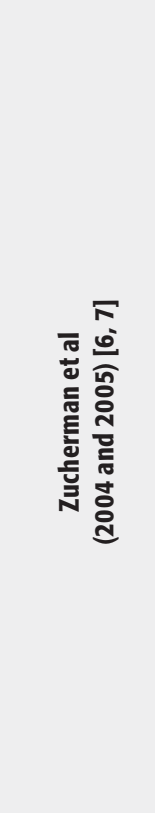 & $\begin{array}{l}\text { Randomized } \\
\text { controlled trial } \\
\text { May 2000-July } \\
2001\end{array}$ & $\begin{array}{l}\text { N= } 191 \\
\text { - Age: } 69.5 \text { y } \\
\text { - Male: NR } \\
\text { Interspinous device } \\
\text { - } \mathrm{n}=100 \\
\text { - Age: } 70 \text { y } \\
\text { - Male: } 57 \% \\
\text { Nonoperative } \\
\text { treatment } \\
\text { - } \mathrm{n}=91 \\
\text { - Age: } 69 \mathrm{y} \\
\text { - Male: } 52 \%\end{array}$ & $\begin{array}{l}\text { Interspinous device: } \\
\text { - Mid-sagittal incision made } \\
\text { over spinous processes of } \\
\text { stenotic levels } \\
\text { - Hypertrophied facets that } \\
\text { blocked entry to anterior } \\
\text { interspinous space trimmed } \\
\text { - XSTOP interspinous device } \\
\text { implanted at one or two levels } \\
\text { Nonoperative: } \\
\text { - Physical therapy consisting of } \\
\text { back school, stabilization } \\
\text { exercises, massage and } \\
\text { modalities } \\
\text { - Received at least one epidural } \\
\text { steroid injection } \\
\text { - Nonsteroidal } \\
\text { antiinflammatories } \\
\text { - Analgesics } \\
\text { - Braces, such as abdominal } \\
\text { binders and corsets, were } \\
\text { permitted }\end{array}$ & $\begin{array}{l}\text { Inclusion: } \\
\text { - X-ray confirmation of LSS } \\
\text { - Leg, buttock, or groin pain with or } \\
\text { without back pain relieved during flexion } \\
\text { - } \geq 50 \text { y } \\
\text { - One or two levels } \\
\text { - Able to sit pain free for } 50 \text { min } \\
\text { - Walk }>50 \text { feet } \\
\text { - Completed } 6 \text { mo of nonoperative } \\
\text { therapy } \\
\text { Exclusion: } \\
\text { - Fixed motor deficit } \\
\text { - Cauda equina syndrome } \\
\text { - Significant lumbar instability } \\
\text { - Previous lumbar surgery } \\
\text { - Significant peripheral neuropathy or } \\
\text { acute denervation secondary to } \\
\text { radiculopathy } \\
\text { - Spondylolisthesis }>\text { grade I } \\
\text { - Sustained pathological fractures } \\
\text { - Severe osteoporosis of vertebrae } \\
\text { - Active infection or systemic disease } \\
\text { - Paget disease or metastasis of vertebrae }\end{array}$ & $\begin{array}{l}24 \text { mo: } \\
\text { - Interspinous device: } \\
93 \% \\
\text { - Nonoperative: 89\% } \\
12 \text { mo: } \\
\text { - Interspinous device: } \\
88 \% \\
\text { - Nonoperative: } 75 \% \\
6 \text { mo: } \\
\text { - Interspinous device: } \\
88 \% \\
\text { - Nonoperative: } 69 \%\end{array}$ \\
\hline
\end{tabular}


Table 2 Clinical outcomes and complications for lumbar spinal stenosis (LSS) treated by decompression or interspinous device.

\begin{tabular}{|c|c|c|c|c|c|}
\hline \multirow{2}{*}{ 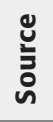 } & \multicolumn{4}{|c|}{ Clinical outcomes } & \multirow[t]{2}{*}{ Other outcomes } \\
\hline & 6 months & 12 months & 24 months & 48 months & \\
\hline 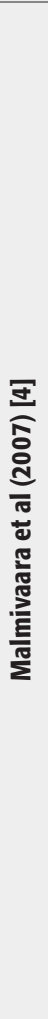 & $\begin{array}{l}\text { Oswestry Disability Index: } \\
\text { - Decomp: -13.3 (34-20.7) } \\
\text { - Nonop: -6.4 (34.7-28.3) } \\
\text { Treatment effect: -6.9 } \\
\text { (95\% Cl: 1.3-13.9) } \\
\text { Numeric Rating Scale for leg } \\
\text { pain during walking: } \\
\text { - Decomp: -4.01 } \\
\text { (6.58-2.57) } \\
\text { - Nonop: -1.71 (6.3-4.59) } \\
\text { Treatment effect: -2.30 } \\
\text { (95\% Cl: 0.69-3.36) } \\
\text { Numeric Rating Scale for low } \\
\text { back pain during walking: } \\
\text { - Decomp: -4.09 (6.9-2.81) } \\
\text { - Nonop: -1.38 (6.83-5.45) } \\
\text { Treatment effect: -2.71 } \\
\text { (95\% Cl: 1.40-3.88) } \\
\text { Increase in walking distance, } \\
\text { meters: } \\
\text { - Decomp: 1503 } \\
\text { (1321-2824) } \\
\text { - Nonop: 1274 (1321-2638) } \\
\text { Treatment effect : 229 } \\
\text { (95\% Cl: -1394-1022) }\end{array}$ & $\begin{array}{l}\text { Oswestry Disability Index: } \\
\text { - Decomp: -15.1 (34-19) } \\
\text { - Nonop: -4.5 (34.7-30.2) } \\
\text { Treatment effect: -10.6 } \\
\text { (95\% Cl: 4.3-18.4) } \\
\text { Numeric Rating Scale for leg } \\
\text { pain during walking: } \\
\text { - Decomp: -3.85 } \\
\text { (6.58- 2.73) } \\
\text { - Nonop: -1.89 (6.3- 4.41) } \\
\text { Treatment effect: -1.96 } \\
\text { (95\% Cl: 0.41-2.96) } \\
\text { Numeric Rating Scale for low } \\
\text { back pain during walking: } \\
\text { - Decomp: -4.18 (6.9-2.72) } \\
\text { - Nonop: -1.78 (6.83-5.05) } \\
\text { Treatment effect: -2.40 } \\
\text { (95\% Cl: 1.12-3.55) } \\
\text { Increase in walking distance, } \\
\text { meters: } \\
\text { - Decomp: 1721 } \\
\text { (1321-3042) } \\
\text { - Nonop: 1460 (1364-2824) } \\
\text { Treatment effect : 261 } \\
\text { Improvement: 130\% } \\
\text { (95\% Cl: -1523-1087) }\end{array}$ & $\begin{array}{l}\text { Oswestry Disability Index: } \\
\text { - Decomp: -12.8 (34-21.2) } \\
\text { - Nonop: -5.7 (34.7-29) } \\
\text { Treatment effect: -7.1 } \\
\text { (95\% Cl: 0.8-14.9) } \\
\text { Numeric Rating Scale for leg } \\
\text { pain during walking: } \\
\text { - Decomp: -3.54 } \\
\text { (6.58- 3.04) } \\
\text { - Nonop: -1.75 (6.3- 4.55) } \\
\text { Treatment effect: -1.79 } \\
\text { (95\% Cl: 0.25-2.77) } \\
\text { Numeric Rating Scale for low } \\
\text { back pain during walking: } \\
\text { - Decomp: -4.16 (6.9-2.74) } \\
\text { - Nonop: -1.96 (6.83- 4.87) } \\
\text { Treatment effect: -2.20 } \\
\text { (95\% Cl: 0.98-3.28) } \\
\text { Increase in walking distance, } \\
\text { meters: } \\
\text { - Decomp: 1508 } \\
\text { (1321-2829) } \\
\text { - Nonop: 1414 (1364-2778) } \\
\text { Treatment effect : 94 } \\
\text { Improvement: 114\% } \\
\text { (95\% Cl: -1338-1235) }\end{array}$ & Not reported & $\begin{array}{l}\text { Complications: } \\
\text { - } 8 \text { perioperative complications including } \\
7 \text { lesions to dural sac corrected with } \\
\text { sutures and } 1 \text { misplaced transpedicular } \\
\text { screw replaced during original surgery } \\
\text { - } 1 \text { postoperative complication of } \\
\text { respiratory distress due to pulmonary } \\
\text { edema combined with stress ulcer } \\
\text { Revision/additional surgery: } \\
\text { - } 4 \text { (9\%) patients in nonoperative group } \\
\text { required surgery because of } \\
\text { exacerbation of symptoms } \\
\text { - } 3 \text { (6\%) further surgeries in } \\
\text { decompression group; } 1 \text { as a result of } \\
\text { misjudgment of stenotic level corrected } \\
\text { same day; } 1 \text { due to peridural hematoma } \\
\text { at } 3 \text { days; } 1 \text { patient had a new } \\
\text { decompressive procedure at } \\
12 \text { mo }\end{array}$ \\
\hline
\end{tabular}


Table 2 (Cont.)

\begin{tabular}{|c|c|c|c|c|c|}
\hline \multirow{2}{*}{$\begin{array}{l}\text { ํㅗㄹ } \\
\text { ํํ }\end{array}$} & \multicolumn{4}{|c|}{ Clinical outcomes } & \multirow[t]{2}{*}{ Other outcomes } \\
\hline & 6 months & 12 months & 24 months & 48 months & \\
\hline \multirow{13}{*}{ 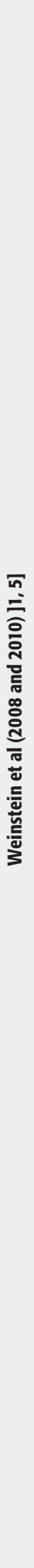 } & $\begin{array}{l}\text { Oswestry Disability Index: } \\
\text { Mean change } \\
\text { - Decomp: }-14.6 \pm 1.9 \\
\text { - Nonop: }-13.7 \pm 1.7 \\
\text { Treatment effect: }-0.9 \\
\text { (95\% Cl: }-5.9-4.1)\end{array}$ & $\begin{array}{l}\text { Oswestry Disability Index: } \\
\text { Mean change } \\
\text { - Decomp: }-14.9 \pm 1.9 \\
\text { - Nonop: }-12.7 \pm 1.8 \\
\text { Treatment effect: }-2.2 \\
\text { (95\% Cl: }-7.4-2.9)\end{array}$ & $\begin{array}{l}\text { Oswestry Disability Index: } \\
\text { Mean change } \\
\text { - Decomp: }-16.4 \pm 1.9 \\
\text { - Nonop: }-12.9 \pm 1.8 \\
\text { Treatment effect: }-3.5 \\
\text { (95\% Cl: }-8.7-1.7)\end{array}$ & $\begin{array}{l}\text { Oswestry Disability Index: } \\
\text { Mean change } \\
\text { - Decomp: }-12.2 \pm 2.0 \\
\text { - Nonop: }-12.4 \pm 1.9 \\
\text { Treatment effect: } 0.2 \\
\text { (95\% Cl: } 5.2-5.7 \text { ) }\end{array}$ & \multirow{13}{*}{ 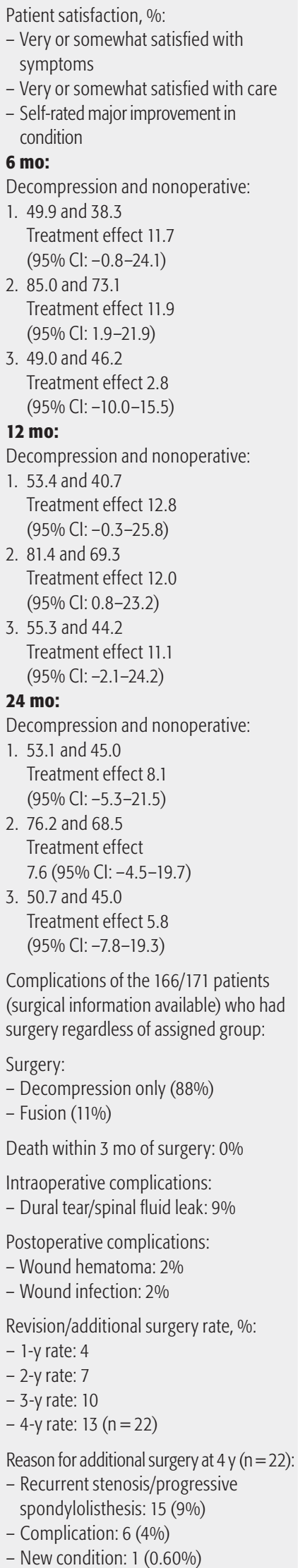 } \\
\hline & $\begin{array}{l}\text { SF-36 Bodily pain: } \\
\text { Mean change } \\
\text { - Decomp: } 21.0 \pm 2.2 \\
\text { - Nonop: } 16.1 \pm 2.1 \\
\text { Treatment effect: } 4.9 \\
\text { (95\% Cl: }-1.2-10.9)\end{array}$ & $\begin{array}{l}\text { SF-36 Bodily pain: } \\
\text { Mean change } \\
\text { - Decomp: } 23.0 \pm 2.3 \\
\text { - Nonop: } 17.5 \pm 2.2 \\
\text { Treatment effect: } 5.5 \\
(95 \% \text { Cl: }-0.7-11.7)\end{array}$ & $\begin{array}{l}\text { SF-36 Bodily pain: } \\
\text { Mean change } \\
\text { - Decomp: } 23.4 \pm 2.3 \\
\text { - Nonop: } 15.6 \pm 2.2 \\
\text { Treatment effect: } 7.8 \\
\text { (95\% Cl: } 1.5-14.1)\end{array}$ & $\begin{array}{l}\text { SF-36 Bodily pain: } \\
\text { Mean change } \\
\text { - Decomp: } 15.9 \pm 2.4 \\
\text { - Nonop: } 15.7 \pm 2.4 \\
\text { Treatment effect: } 0.3 \\
(95 \% \text { Cl: }-6.4-7.0)\end{array}$ & \\
\hline & $\begin{array}{l}\text { SF-36 Physical function: } \\
\text { Mean change } \\
\text { - Decomp: } 17.6 \pm 2.3 \\
\text { - Nonop:15.1 } \pm 2.2 \\
\text { Treatment effect: } 2.5 \\
\text { (95\% Cl: }-3.7-8.6)\end{array}$ & $\begin{array}{l}\text { SF-36 Physical function: } \\
\text { Mean change } \\
\text { - Decomp: } 18.0 \pm 2.3 \\
\text { - Nonop: } 16.4 \pm 2.2 \\
\text { Treatment effect: } 1.6 \\
(95 \% \text { Cl: }-4.8-7.9)\end{array}$ & $\begin{array}{l}\text { SF-36 Physical function: } \\
\text { Mean change } \\
\text { - Decomp: } 17.1 \pm 2.4 \\
\text { - Nonop: } 17.1 \pm 2.3 \\
\text { Treatment effect: } 0.1 \\
\text { (95\% Cl: }-6.4-6.5)\end{array}$ & $\begin{array}{l}\text { SF-36 Physical function: } \\
\text { Mean change } \\
\text { - Decomp: } 12.7 \pm 2.5 \\
\text { - Nonop: } 15.9 \pm 2.4 \\
\text { Treatment effect: }-3.2 \\
\text { (95\% Cl: }-9.9-3.6)\end{array}$ & \\
\hline & $\begin{array}{l}\text { Stenosis Bothersome Index: } \\
\text { NR } \\
\text { Leg Pain Bothersome Index: } \\
\text { NR } \\
\text { Low Back Pain Bothersome }\end{array}$ & $\begin{array}{l}\text { Stenosis Bothersome Index: } \\
\text { Mean change } \\
\text { - Decomp: }-6.1 \pm 0.7 \\
\text { - Nonop: }-4.9 \pm 0.7 \\
\text { Treatment effect: }-1.2 \\
(95 \% \text { Cl: }-3.2-0.8)\end{array}$ & $\begin{array}{l}\text { Stenosis Bothersome Index: } \\
\text { Mean change } \\
\text { - Decomp: }-6.3 \pm 0.7 \\
\text { - Nonop: }-5.6 \pm 0.7 \\
\text { Treatment effect: }-0.7 \\
(95 \% \text { Cl: }-2.7-1.3)\end{array}$ & $\begin{array}{l}\text { Stenosis Bothersome Index: } \\
\text { Mean change } \\
\text { - Decomp: }-5.2 \pm 0.75 \\
\text { - Nonop: }-4.5 \pm 0.73 \\
\text { Treatment effect: }-0.7 \\
(95 \% \text { Cl: }-2.8-1.4)\end{array}$ & \\
\hline & & $\begin{array}{l}\text { Leg Pain Bothersome Index: } \\
\text { Mean change } \\
\text { - Decomp: }-2.3 \pm 0.2 \\
\text { - Nonop: }-1.7 \pm 0.2 \\
\text { Treatment effect: }-0.6 \\
(95 \% \text { Cl: }-1.3-0)\end{array}$ & $\begin{array}{l}\text { Leg Pain Bothersome Index: } \\
\text { Mean change } \\
\text { - Decomp: }-2.2 \pm 0.2 \\
\text { - Nonop: }-1.8 \pm 0.2 \\
\text { Treatment effect: }-0.3 \\
(95 \% \text { Cl: }-1.0-0.3)\end{array}$ & $\begin{array}{l}\text { Leg Pain Bothersome Index: } \\
\text { Mean change } \\
\text { - Decomp: }-1.8 \pm 0.2 \\
\text { - Nonop: }-1.8 \pm 0.2 \\
\text { Treatment effect: } 0 \\
(95 \% \text { Cl: }-0.7-0.6)\end{array}$ & \\
\hline & & $\begin{array}{l}\text { Low Back Pain Bothersome } \\
\text { Index: } \\
\text { Mean change } \\
\text { - Decomp: }-1.3 \pm 0.2 \\
\text { - Nonop: }-1.3 \pm 0.2 \\
\text { Treatment effect: } 0 \\
(95 \% \text { Cl: }-0.5-0.6)\end{array}$ & $\begin{array}{l}\text { Low Back Pain Bothersome } \\
\text { Index: } \\
\text { Mean change } \\
\text { - Decomp: }-1.3 \pm 0.2 \\
\text { - Nonop: }-1.6 \pm 0.2 \\
\text { Treatment effect: }-0.3 \\
(95 \% \text { Cl: }-0.2-0.9)\end{array}$ & $\begin{array}{l}\text { Low Back Pain Bothersome } \\
\text { Index: } \\
\text { Mean change } \\
\text { - Decomp: }-0.9 \pm 0.2 \\
\text { - Nonop: }-1.3 \pm 0.2 \\
\text { Treatment effect: } 0.4 \\
(95 \% \text { Cl: }-0.2-1.0)\end{array}$ & \\
\hline & & & & & \\
\hline & & & & & \\
\hline & & & & & \\
\hline & & & & & \\
\hline & & & & & \\
\hline & & & & & \\
\hline & & & & & \\
\hline
\end{tabular}


Table 2 (Cont.)

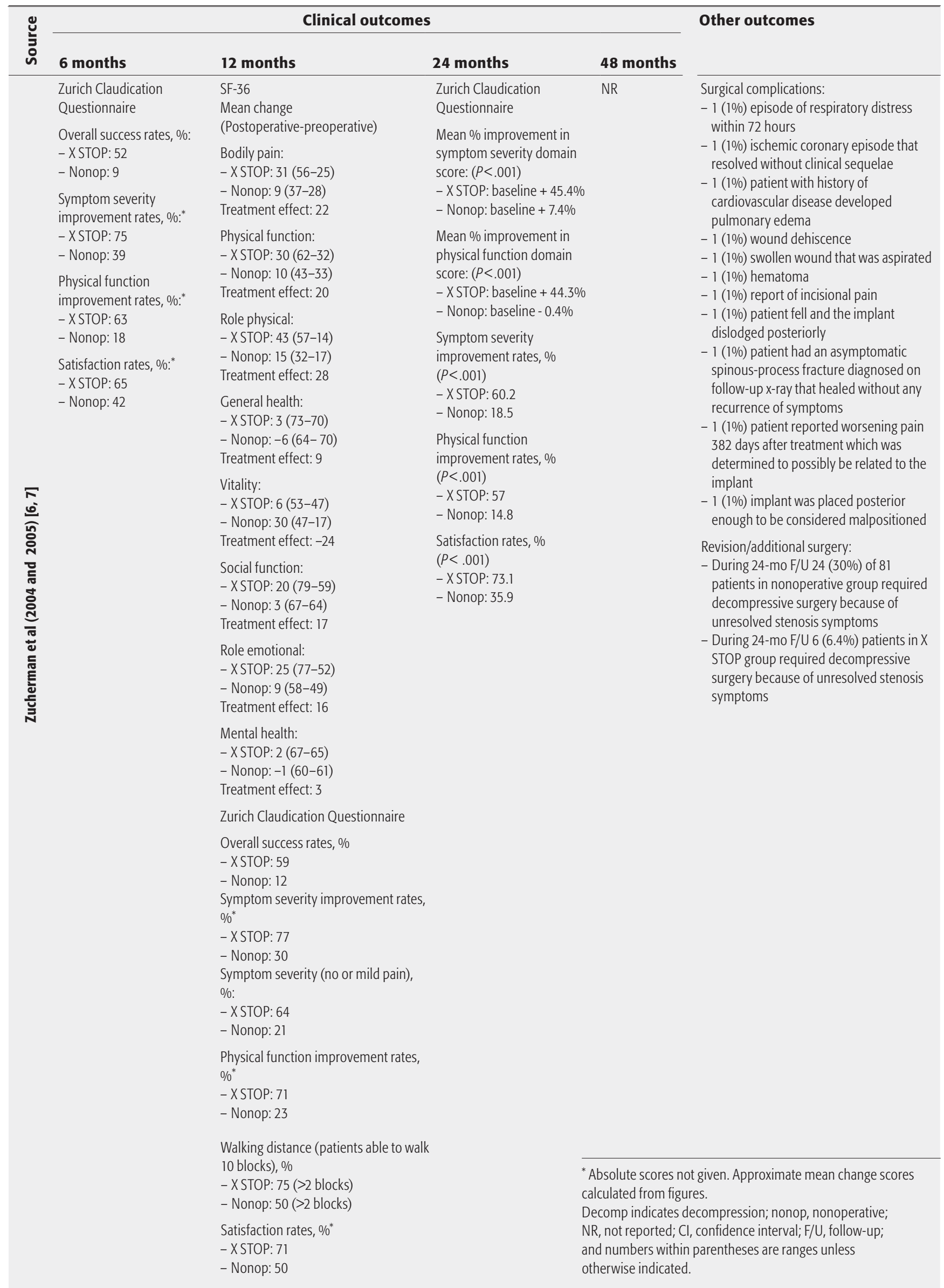


Table 3 Changes from baseline to 12 and 24 months postoperatively and corresponding treatment effects for indirect comparisons of each surgical arm to a conservative arm.

\begin{tabular}{|c|c|c|c|c|c|c|c|c|c|}
\hline \multirow[t]{2}{*}{ Outcome } & \multirow[t]{2}{*}{ Reference } & \multicolumn{2}{|c|}{ Patients, N } & \multicolumn{2}{|c|}{ Interspinous device } & \multicolumn{2}{|c|}{ Decompression } & \multicolumn{2}{|c|}{$\begin{array}{l}\text { Treatment effect } \\
\text { A or B vs C }\end{array}$} \\
\hline & & Total & $\operatorname{Rx} \operatorname{arm}(s)$ & 12-mo change & 24-mo change & 12-mo change & 24-mo change & $12 \mathrm{mo}$ & $24 \mathrm{mo}$ \\
\hline \multirow{2}{*}{$\begin{array}{l}\text { Disability } \\
\text { SF-36 Physical Function }\end{array}$} & Weinstein et al [1, 5] & 289 & $B(138)$ & - & - & 18.0 & 17.1 & 1.6 & 0.1 \\
\hline & Zucherman et al $[6,7]$ & 191 & $A(100)$ & 30 & NR & - & - & 20 & NR \\
\hline \multirow{2}{*}{$\begin{array}{l}\text { Pain } \\
\text { SF-36 Bodily Pain }\end{array}$} & Weinstein et al $[1,5]$ & 289 & B (138) & - & - & 23.0 & 23.4 & 5.5 & 7.8 \\
\hline & Zucherman et al $[6,7]$ & 191 & $A(100)$ & 31 & NR & - & - & 22 & NR \\
\hline \multirow{2}{*}{$\begin{array}{l}\text { Function } \\
\text { Walking distance }\end{array}$} & Malmivaara et al [4] & 94 & $B(50)$ & - & - & $130 \%$ & $114 \%$ & $23 \%$ & $10 \%$ \\
\hline & Zucherman et al $[6,7]$ & 191 & $A(100)$ & $75 \%$ & NR & - & - & $25 \%$ & NR \\
\hline \multirow{3}{*}{$\begin{array}{l}\text { Safety } \\
\text { Complication rates* }\end{array}$} & Malmivaara et al [4] & 94 & $B(50)$ & - & - & $27 \%$ & NR & $27 \%$ & NR \\
\hline & Weinstein et al $[1,5]$ & 289 & $B(138)$ & - & - & $11.6 \%$ & NR & $11.6 \%$ & 0 \\
\hline & Zucherman et al $[6,7]$ & 191 & $A(100)$ & $11 \%$ & NR & - & - & $11 \%$ & 0 \\
\hline
\end{tabular}

Dashes represent treatment arms that were not included in the particular study. NR indicates not reported; Rx, treatment arms ( $A$, interspinous device; $\mathrm{B}$, decompression; C, conservative); and N, all subjects in study. Change in points at 12 months and 24 months if no units are listed; change is given in percentage. The lower the score, the higher the function.

Treatment effect indicates difference between interspinous (A) or decompression (B) versus conservative (C) arm change scores.

* All postoperative complications, except additional surgery.

Table 4 Comparing 12- and 24-month mean percentage improvements in Zurich Claudication subscale scores and treatment effects between interspinous device and conservative management in the study by Zucherman et al $[6,7]$.

\begin{tabular}{|c|c|c|c|c|c|c|}
\hline \multirow[t]{2}{*}{ Improvement } & \multicolumn{2}{|c|}{ Interspinous } & \multicolumn{2}{|c|}{ Conservative } & \multicolumn{2}{|c|}{ Treatment effect } \\
\hline & 12 months & 24 months & 12 months & 24 months & 12 months & 24 months \\
\hline Physical function, \% & 71 & 57 & 23 & 14.8 & 48 & 42.2 \\
\hline Symptom severity, \% & 77 & 60.2 & 30 & 18.5 & 47 & 47.2 \\
\hline
\end{tabular}

Table 5 Comparing 12- and 24-month improvements in the mean Oswestry Disability Index (ODI) scores and treatment effects between decompression and conservative management in the studies by Malmivaara et al [4] and Weinstein et al [1,5], respectively.

\begin{tabular}{|c|c|c|c|c|c|c|}
\hline \multirow[t]{2}{*}{ Score } & \multicolumn{2}{|c|}{ Decompression } & \multicolumn{2}{|c|}{ Conservative } & \multicolumn{2}{|c|}{ Treatment effect } \\
\hline & 12 months & 24 months & 12 months & 24 months & 12 months & 24 months \\
\hline ODI (points) & -15.1 & -12.8 & -4.5 & -5.7 & -10.6 & -7.1 \\
\hline ODI (points) & -14.9 & -16.4 & -12.7 & -12.9 & -2.2 & -3.5 \\
\hline
\end{tabular}

Negative scores represent improvement in function. 
Fig 1 Results of literature search.

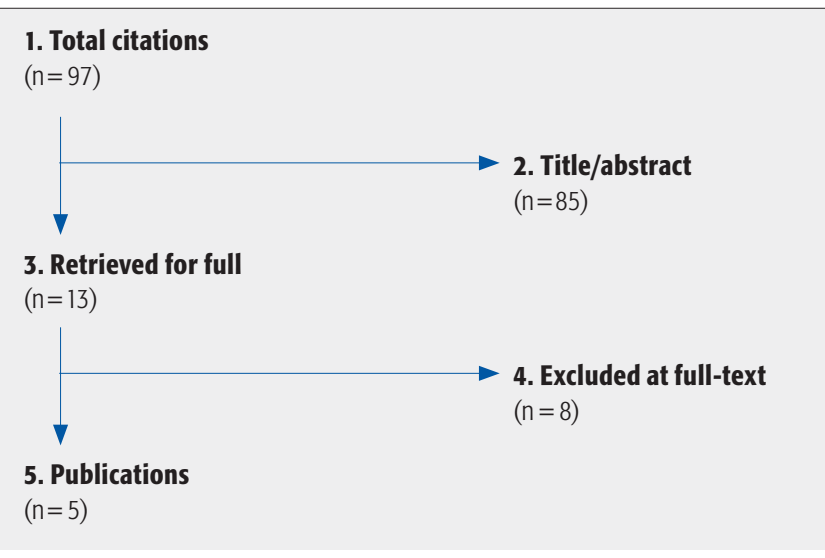

Fig 2 Indirect treatment effects (TE) ${ }^{*}$ comparing surgical

decompression to interspinous-device placement.

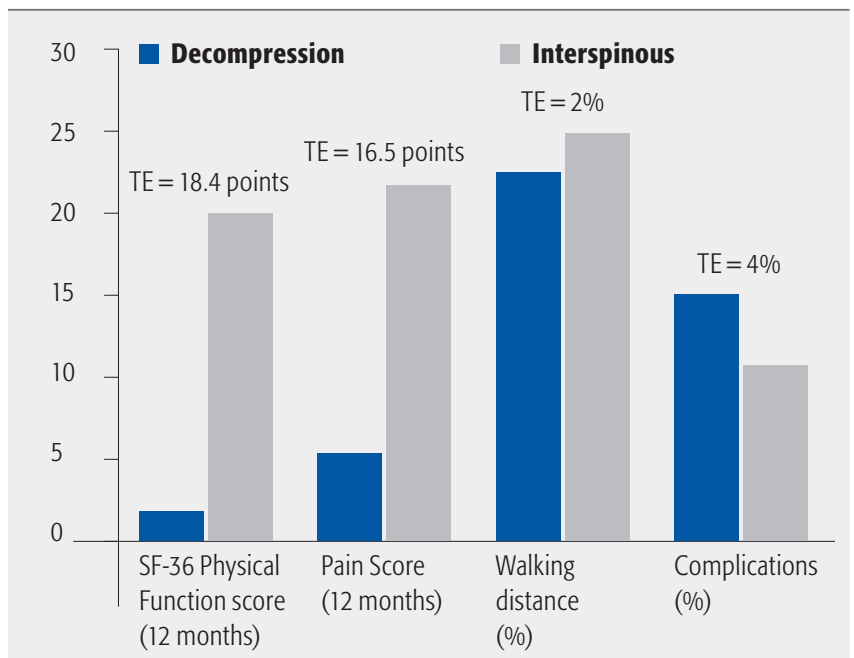

${ }^{*}$ Indirect treatment effects are based on the difference between decompression versus conservative management and interspinous-device placement versus conservative management.

\section{EVIDENCE SUMMARY}

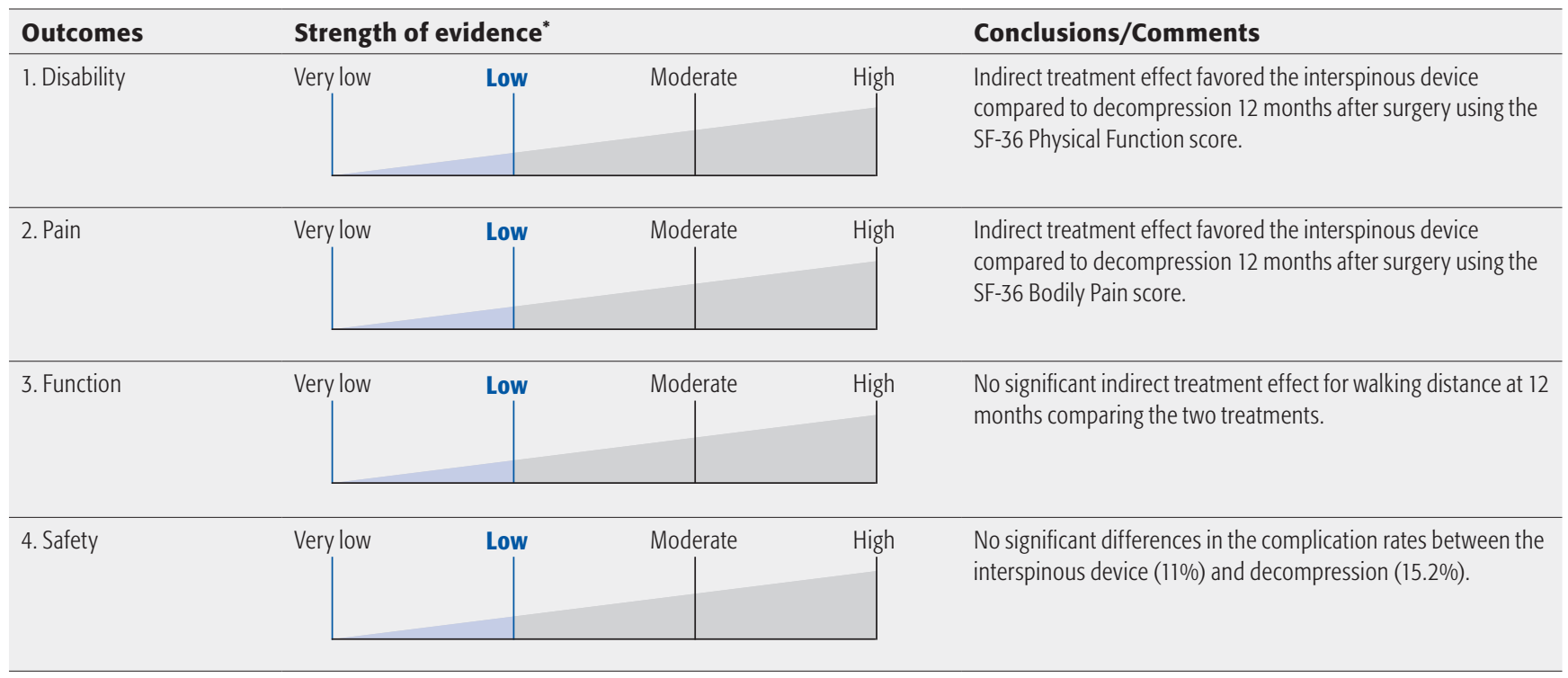

* Low evidence indicates further research is likely to have an important impact on the confidence in the estimate of effect and is likely to change the estimate. 


\section{DISCUSSION}

There was low evidence supporting greater treatment effects for interspinous-device placement compared to decompression for disability and pain outcomes at 12 months. There was low evidence demonstrating little to no difference in treatment effects between groups for walking distance and complication rates. GRADE criteria suggest that low evidence indicates "further research is very likely to have an important impact on the confidence in the estimate of effect and is likely to change the estimate."

The observations that are seen in this modified network analysis must be taken within the context that no direct comparisons between interspinous spacers and decompressive laminectomy exist. Thus, the network analysis model analyzes best available evidence from the two different treatment studies for an indirect comparison.

This indirect comparison must be carefully analyzed because of the inherent possibilities of different treatment groups (ie, heterogeneity).

The first caveat is the intent-to-treat analysis as opposed to an as-treated analysis. In the Weinstein et al study $[1,5], 43 \%$ of the patients who were in the nonsurgical arm crossed over to having surgery at the 2-year mark. In addition, only $67 \%$ of the surgical arm had actually undergone surgery. In the article, the authors' state, “ ... the intention-to-treat analysis underestimates the true effect of surgery." They go on to conclude, "In the as-treated analysis ... those treated surgically showed significantly greater improvement ... " Further, none of the patients in Zucherman et al [6, 7] crossed over. Thus, because of the cross over in the Weinstein et al study [1,5], the treatment effect may not be as great as that seen in the Zucherman et al study [6, 7].
Another point to consider is the difference in the enrollment of patients. Weinstein et al $[1,5]$ and Malmivaara et al [4] enrolled patients who were diagnosed with lumbar stenosis who failed conservative therapy. Zucherman et al $[6,7]$, however, enrolled patients who had pain relief while sitting. Thus, the patients enrolled in that study were known to have alleviation of pain with their lumbar spines in flexion. This may have created a selection bias in that this study only enrolled patients who would respond favorably to an interspinous device.

Morbidity was calculated from the complication rates, both perioperatively and postoperatively. Note that both lumbar decompressive surgery groups (Weinstein et al [1,5] and Malmivaara et al [4]) had patients with instrumented fusion (a more morbid procedure than decompression alone). This would have skewed the complication rate higher for the surgical decompression groups, since these were not simple decompressive procedures.

The indirect treatment effect for disability and pain favors the interspinous device compared to decompression. No significant treatment effect differences were observed for postoperative-walking distance improvement or complication rates; however, findings should be considered with caution due to indirect comparisons and short follow-up periods.

Given the limitations of our network analysis, we nonetheless evaluated the best evidence currently available. Eventually, studies with direct comparisons evaluating the efficacy between interspinous devices and surgical decompression will hopefully give a more precise answer. 


\section{REFERENCES}

1. Weinstein JN, Tosteson TD, Lurie JD, et al (2008) Surgical versus nonsurgical therapy for lumbar spinal stenosis. $N$ Engl $J$ Med; 358(8):794-810.

2. Richter A, Schütz C, Hauck M, et al (2010) Does an interspinous device (Coflex) improve the outcome of decompressive surgery in lumbar spinal stenosis? One-year follow up of a prospective case control study of 60 patients. Eur Spine J; 19(2):283-289.

3. Park SC, Yoon SH, Hong YP, et al (2009) Minimum 2-year follow-up result of degenerative spinal stenosis treated with interspinous u (coflex). J Korean Neurosurg Soc; 46(4):292-299.

4. Malmivaara A, Slätis $\mathbf{P}$, Heliövaara $\mathbf{M}$, et al (2007) Surgical or nonoperative treatment for lumbar spinal stenosis? A randomized controlled trial. Spine; 32(1):1-8.

5. Weinstein JN, Tosteson TD, Lurie JD, et al (2010) Surgical versus nonoperative treatment for lumbar spinal stenosis four-year results of the Spine Patient Outcomes Research Trial. Spine; 35(14):1329-1338.

6. Zucherman JF, Hsu KY, Hartjen CA, et al (2004) A prospective randomized multi-center study for the treatment of lumbar spinal stenosis with the X STOP interspinous implant: 1-year results. Eur Spine J; 13(1):22-31.

7. Zucherman JF, Hsu KY, Hartjen CA, et al (2005) A multicenter, prospective, randomized trial evaluating the X STOP interspinous process decompression system for the treatment of neurogenic intermittent claudication: two-year follow-up results. Spine; 30(12):1351-1358.

\section{EDITORIAL STAFF PERSPECTIVE}

There are several noteworthy factors regarding this topic and the systematic review as performed:

- The current strength of evidence favoring interspinous spacers compared to decompression surgery with or without fusion regarding factors of disability and pain is low, meaning that "Further research is very likely to have an important impact on our confidence in the estimate of effect and is likely to change the estimate."

- There was general surprise voiced that there is no direct comparison study between decompression surgery alone (in properly selected patients) and interspinous spacers without decompression. For various reasons the higher-grade evidence relies on comparisons, such as interspinous spacers versus nonoperative modalities, decompression and fusion versus decompression and surgical decompression versus nonoperative treatment. The absence of the most compelling form of comparison studies - decompression alone versus interspinous spacer for more stable forms of stenosis and decompression and fusion versus decompression and interspinous spacer for more unstable forms of stenosis-was quoted as being "lamentable" and "overdue for correction."

- The absence of clinically relevant direct comparison studies necessitated a 'network analysis' with its inherent shortcomings of error through heterogeneity of its study cohorts and lower level of familiarity to a clinician community.

- The short-term follow-up of studies with interspinous spacers (12 months) was noted to likely underreport complications, such as device loosening and need for more complex revision surgery for patients with interspinous devices, which would appear to be more likely to fail with time.

- The impact of patients' complications with interspinous devices as expressed in invasiveness of potential corrective surgery compared to less expensive and simple decompression surgery is presently not understood.

- The potential for financial conflict of interest affecting several authors related to the development and subsequent marketing of certain interspinous devices was pointed out. To date there are no prospective comparison studies from financially disinterested third-party groups. 


\section{Case example \\ (Case provided by Jens Chapman)}

Two years after L3/4 interspinous process-spacer placement a physically very active and healthy 65-year-old man presented with severe bilateral leg pain brought on by short-distance walking and relieved by bending and squatting (Fig 3a-c). At the time the procedure had brought substantial symptom relief, however progressive symptom recurrence was noted about 18 months after the index procedure. The patient was found to have an X-Stop device at his L3/4 interspace with heterotopic bone formation surrounding the implant, as well as a subtle grade 1 degenerative spondylolisthesis.

The MRI scan revealed persistent significant central stenosis with facet hypertrophy and lateral recess compromise (Fig 3d). Because of failure of nonoperative treatment the patient underwent removal of the device and simple midline-sparing hemilaminotomies with partial facetectomies through a keyhole laminotomy approach (Fig 3e). Abundant heterotopic bone surrounding the implant was carefully removed, while avoiding injury to the ligaments. Since decompressive surgery the patient experienced complete relief of lower extremity and back symptoms and return to activities of daily living including competitive golf 6 months to date.

This case illustrates some concerns about interspinous spacers. The role of simple soft-tissue-sparing decompression surgery, while avoiding destabilizing measures, compared to an implantbased nonfusion procedure remains to be established and cannot be concluded based on the current state of the literature. Does a nonfusion device in fact set up patients for more revision surgeries in the intermediate and longer run compared to simple decompression or fusion surgery in appropriately selected patient? Another question worth debating is how long does a satisfactory result in elective spine surgery have to last to be counted as a success? Alternatively, when is a revision procedure performed at an index level a complication? This case certainly illustrates some of the many unanswered questions.
Fig 3a Preoperative x-ray (AP view).

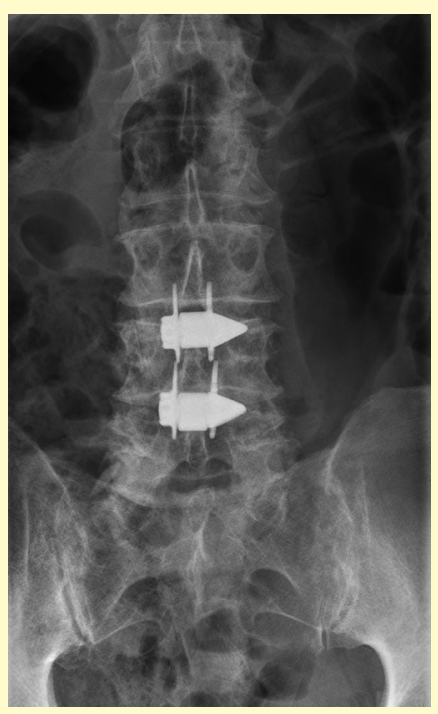

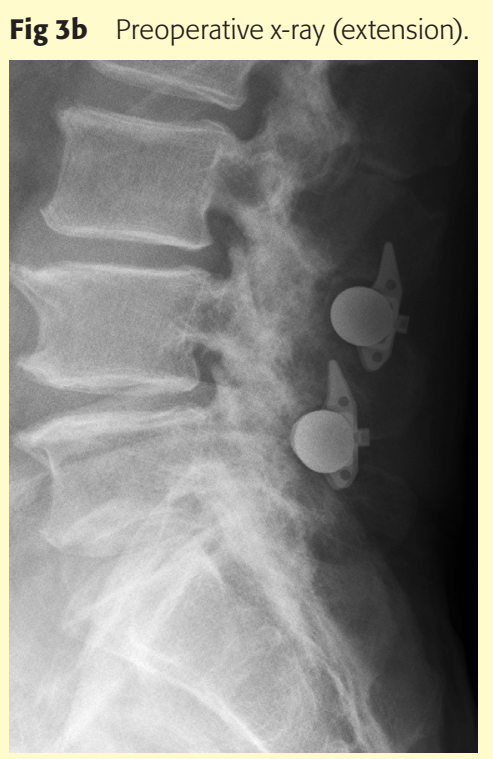

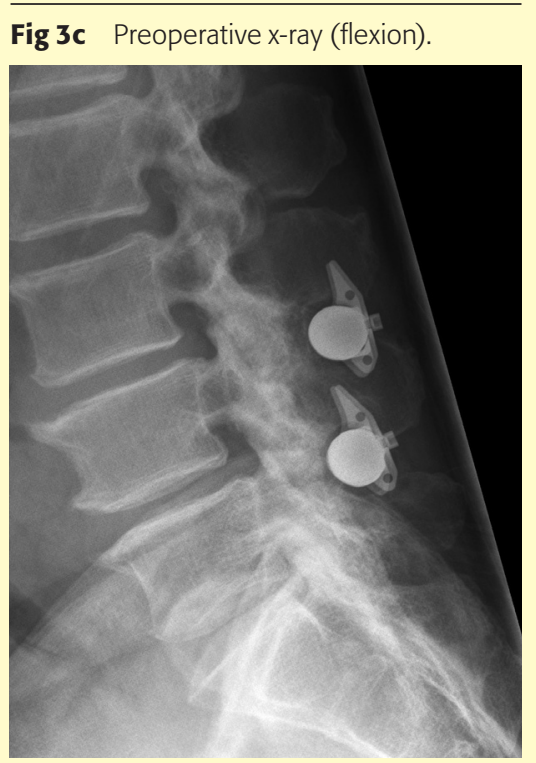

Fig 3d Magnetic resonance imaging (axial).

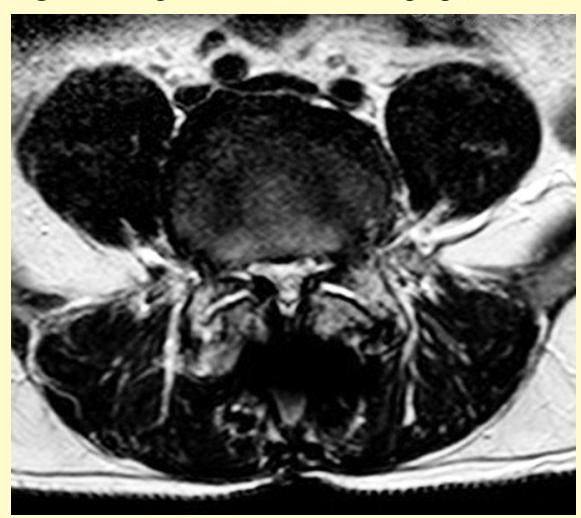

Fig 3e The intraoperative site revealed extensive foreign body debris (dark spots) and abundant reactive connective tissue build up around the area of the interspinous spacers.

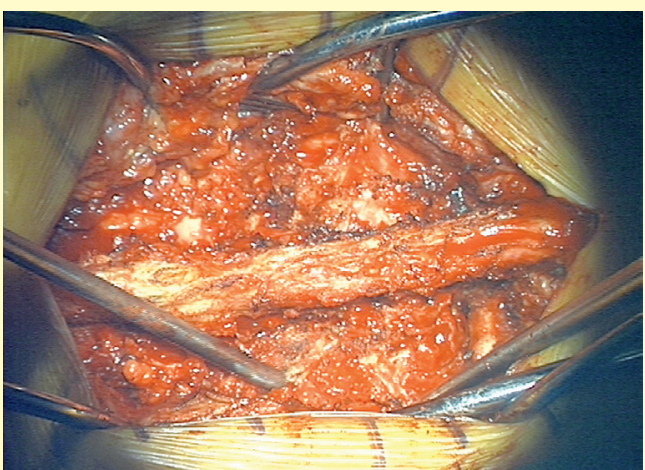

